\title{
Patient's Perception on Leg Length Discrepancy After Total Hip Arthroplasty in Patients with Unilateral Crowe Type IV Developmental Dysplasia of the Hip
}

This article was published in the following Dove Press journal:

Patient Preference and Adherence

Yin-qiao Du (D*
Jun-min Shen*
Jing-yang Sun
Chi Xu (D
Ming Ni
Yong-gang Zhou

Department of Orthopaedics, General Hospital of Chinese People's Liberation Army, Beijing 100853, People's Republic of China

*These authors contributed equally to this work
Correspondence: Ming Ni; Yong-gang Zhou Department of Orthopedics, General Hospital of Chinese People's Liberation Army, 28 Fuxing Road, Haidian District, Beijing, People's Republic of China

Tel +86I358I9|5896; +86I380I287599

Email niming30।@।63.com;

ygzhou30I@I63.com
Purpose: The study assessed the correlation among the patients' perception of leg length discrepancy (LLD) after total hip arthroplasty (THA) in patients with unilateral Crowe type IV developmental dysplasia of the hip (DDH) and the four methods of measuring the leg length in the full-length standing anteroposterior radiographs.

Methods: Sixty patients with unilateral Crowe type IV DDH were recruited in this retrospective study between January 2012 and January 2019. Four methods of measurement were used: 1) TD-TP: distance between the inferior aspect of teardrop (TD) and the midpoint of tibial plafond (TP); 2) CH-TP: distance between the center of the hip (CH) or acetabular cup and the TP; 3) GT-TP: distance between the apex of greater trochanter (GT) and the TP; and 4) FL+TL: the sum of femoral length (FL) and tibial length (TL).

Results: Association was found among the patients' perception on LLD with difference in TD-TP $(\mathrm{OR}=1.157)$, and the difference in $\mathrm{FL}+\mathrm{TL}(\mathrm{OR}=1.166)$. The area under the curve of the difference in FL+TL and the difference TD-TP (0.704 and 0.679) was significantly higher than those of the difference in CH-TP and the difference in GT-TP (0.564 and 0.483). With the calculated threshold of LLD set at $9.0 \mathrm{~mm}$, the sensitivity and specificity of the difference in TD-TP and the difference in FL+TL were $57.7 \%, 79.4 \%$ and $61.5 \%, 79.4 \%$, respectively. Conclusion: Patients' perception on LLD had good correlation and reliability on the difference of FL+TL and the difference of TD-TP on both sides in the full-length standing anteroposterior radiographs after THA in patients with unilateral Crowe type IV DDH. The calculated threshold of the difference in FL+TL and the difference in TD-TP was set at $9.0 \mathrm{~mm}$ to assess the patients' perception on LLD.

Keywords: total hip arthroplasty, developmental dysplasia of the hip, leg length discrepancy, patient's perception

Leg length discrepancy (LLD) is a frequent and serious postoperative complication after total hip arthroplasty (THA) in patients with Crowe type IV developmental dysplasia of the hip $(\mathrm{DDH})^{1,2}$ and its clinical importance is due to its association with increased incidence of gait disorders, chronic back pain, neurological sequelae, and general postoperative dissatisfaction. ${ }^{3}$ In Crowe type IV DDH patients, it is difficult to correct LLD during THA due to the preoperative high hip dislocation. ${ }^{4,5}$ Through the comprehensive preoperative plan and surgical techniques, the surgeon succeeded in correcting the LLD on the radiographs. However, the patients were 
still not satisfied. Therefore, LLD should be divided into radiographic LLD and perceived LLD. Radiographic LLD detected in the full-length standing anteroposterior radiographs is the objective LLD, and the surgeon tends to focus on the importance of radiographic LLD, whereas perceived LLD is subjective LLD, and the patients pay close attention to the significance of their own perception.

In many studies, the distance between the center of hip $(\mathrm{CH})$ and the midpoint of tibial plafond (TP) was used as the leg length (CH-TP). ${ }^{6-8}$ These studies they found that perceived LLD had poor correlation and reliability, low sensitivity, and specificity when compared with radiographic LLD. ${ }^{7,8}$ However, the position of the acetabular shell will affect the length of the leg, and the distance between the center of hip and the midpoint of the tibial plafond does not represent the true functional leg length. Woolson et $\mathrm{al}^{9}$ recommend that the acetabular teardrop could be used as a pelvic reference when measuring LLD because the teardrop was a separate anatomic structure, and has no effect on measurement of pelvic rotation in the vertical position. Therefore, we define the distance between the inferior aspect of teardrop (TD) and the midpoint of tibial plafond (TP) was used as the leg length (TD-TP) in patients with unilateral Crowe type IV. LLD is expressed as the difference of the leg length on both sides.

In this study, four methods of measuring the leg length in the full-length standing anteroposterior radiographs were used, including CH-TP, TD-TP, the distance between the apex of greater trochanter and the midpoint of tibial plafond (GT-TP) and the sum of femoral length (FL) and tibial length (TL). Our hypotheses were that the perceived LLD correlate better with TD-TP and the sum of FL and TL.

\section{Methods}

The hospital's institute review board approved this study. Written informed consent was obtained from all patients for publication of this study and any accompanying images. Between January 2012 and January 2019, 60 patients were recruited in this retrospective study. Patients had THA for Crowe type IV DDH. Excluded from the study were patients 1) with less than 12 months after THA, 2) with a history of surgery for lumbar, pelvis, hip, knee, and lower legs, 3) with severe hip osteoarthritis or DDH of contralateral side, 4) with residual DDH (infection and trauma) of the operative side, 5) with adduction or flexion contracture of the hip, 6) with knee flexion deformity or severe knee osteoarthritis, and 7) with history of cerebral palsy and poliomyelitis.

\section{Surgery}

All THA were performed by a single surgeon (Y. G Zhou) in the lateral decubitus position, with posterolateral approach. The procedure has been described in detail in our previous studies. ${ }^{10-12}$ A cementless acetabular shell that was fixed by two screws was placed at the level of the true acetabulum. If the hip was hard reduction, the shortening subtrochanteric osteotomy (SSTO) was performed. Cerclage wiring (two or three steel wires) was done around the location of the osteotomy to prevent fractures. A sleeve or cone was chosen based on the proximal femoral intramedullary morphology, and the femoral component was selected as the one that best matched the femoral intramedullary canal. The Pinnacle acetabular shell, Biolox delta ceramic liner and femoral head, a S-ROM femoral stem with proximal sleeve or cone (DePuy, Warsaw, IN) was used in all patients.

They were asked about their perception of the leg lengths. We asked patients, "Do you experience any uncomfortable feelings about the length of your legs?" and enable them to choose one of three answers: "comfortable," "uncomfortable because I feel the operative leg is too short," and "uncomfortable because I feel the operative leg is too long". 13

All patients were evaluated using the full-length standing anteroposterior radiographs. Each leg was internally rotated $\left(15^{\circ}\right)$ to ensure that the patella was located anteriorly. A Revolution XR/d digital imaging system (GE Healthcare, Milwaukee, WI) with the standard radiographic procedure was used. Six images were generated to acquire a full-length standing anteroposterior radiograph of the lower extremities. All images were combined into a whole limb image. All the radiographs were viewed and measured on a picture archiving and communication system (PACS, UniWeb Viewer, version 4.0, EBM technologies).

We used the following definitions for measuring the length in the full-length standing anteroposterior radiographs (Figure 1): 1) TD-TP: distance between the inferior aspect of teardrop (TD) and the midpoint of tibial plafond (TP). 2) CH-TP: distance between the center of hip (CH) and the midpoint of tibial plafond (TP). 3) GT-TP: distance between the apex of greater trochanter (GT) and the midpoint of tibial plafond (TP). 4) Femoral length (FL): distance between the inferior aspect of teardrop and the articular surface of medial femoral condyle. 5) Tibial length (TL): distance between the center of the tibial 


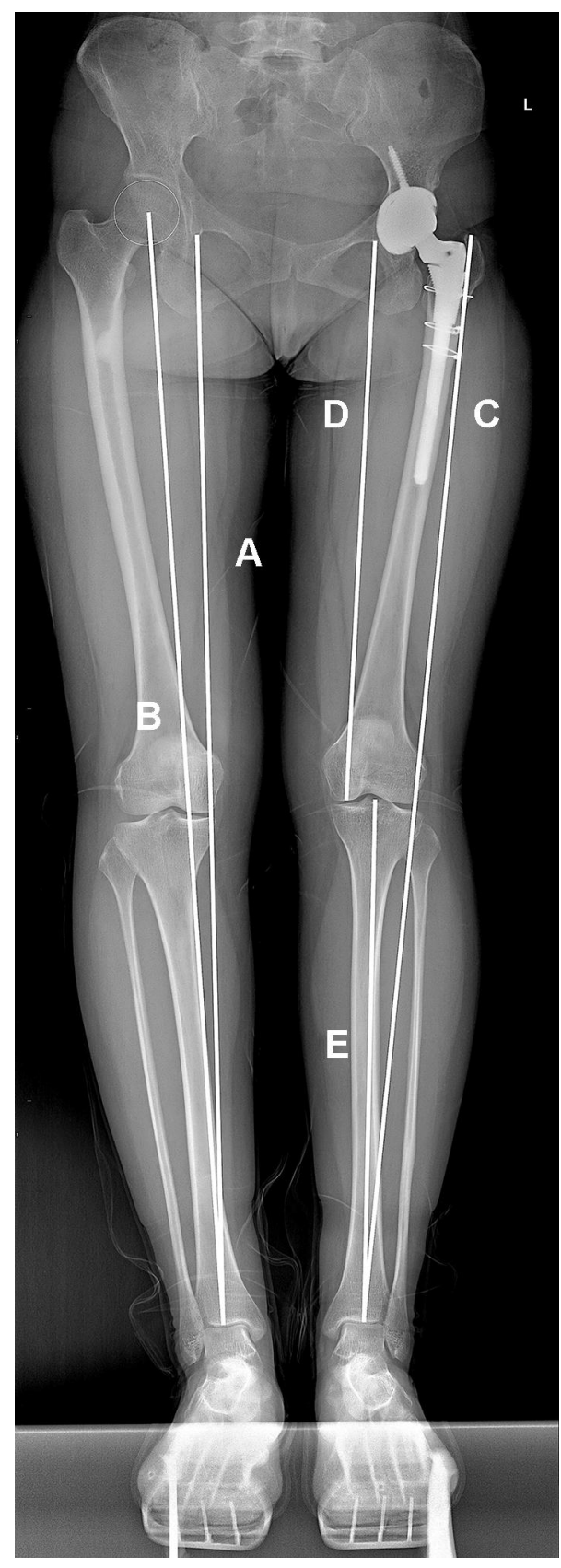

Figure I The full-length standing anteroposterior radiograph. (A) TD-TP: distance between the inferior aspect of teardrop (TD) and the midpoint of tibial plafond (TP). (B) CH-TP: distance between the center of hip $(\mathrm{CH})$ or acetabular cup and the midpoint of tibial plafond. (C) GT-TP: distance between the apex of greater trochanter (GT) and the midpoint of tibial plafond. (D) femoral length (FL): distance between the inferior aspect of teardrop and the articular surface of medial femoral condyle. (E) tibial length (TL): distance between the center of the tibial intercondylar eminence and the midpoint of tibial plafond.

intercondylar eminence and the midpoint of tibial plafond. The leg length of sectional measurement is the sum of FL and TL (FL+TL).

The actual value for each measurement was obtained by calibration using the known diameter of the ceramic femoral head. Two independent investigators (YQD and JMS), who were already trained in PACS measurement, performed the radiographic measurements. The mean of both measurements was the final value. All of the measurements were repeated 4 weeks later to assess the intra-observer reliability.

\section{Statistical analysis}

The intraclass correlation coefficient (ICC) was used to determine the variations of the different measurements. Categorical data were compared using a chi-squared test. The paired samples $t$-test or Wilcoxon test was used to compare continuous data between the operative and contralateral sides. The independent-samples $t$-test or MannWhitney $U$-test was used to compare continuous data between the perception and no perception on LLD. Multiple logistic regression was used to investigate the correlation between the radiographic LLD and perceived LLD. Receiver operating characteristic (ROC) curves were generated to determine the value of each measurement for radiographic LLD. All tests were performed using SPSS version 26 (IBM Corp., Armonk, NY) for Mac. A $P$-value $<0.05$ was considered significant in all analyses.

\section{Results}

The detailed data of patients are presented in Table 1. The differences in FL, TL, TD-TP, CH-TP, GT-TP, and FL+TL are presented in Table 2 . The statistically significant differences were identified in the difference of TD-TP $(P=0.018)$ and the difference of FL+TL $(P=0.009)$

Table I Patient Characteristics

\begin{tabular}{|c|c|c|c|}
\hline Variables & $\begin{array}{l}\text { No Perception on } \\
\text { LLD }\end{array}$ & $\begin{array}{l}\text { Perception on } \\
\text { LLD }\end{array}$ & $P$-values \\
\hline Patients (n) & 34 & 26 & \\
\hline Gender (n) & & & 0.444 \\
\hline Male & 3 & I & \\
\hline Female & 31 & 25 & \\
\hline Age (years) & $37.7 \pm I I .7$ & $40.8 \pm 10.9$ & 0.289 \\
\hline Height $(\mathrm{cm})$ & $159.2 \pm 6.7$ & $|59.4 \pm 5|$. & 0.915 \\
\hline Weight (kg) & $55.8 \pm 9.9$ & $59.3 \pm 11.4$ & 0.208 \\
\hline BMI $\left(\mathrm{kg} / \mathrm{m}^{2}\right)$ & $22.0 \pm 3.2$ & $23.2 \pm 3.6$ & 0.151 \\
\hline Side (n) & & & 0.330 \\
\hline Right & 14 & 14 & \\
\hline Left & 20 & 12 & \\
\hline
\end{tabular}

Abbreviations: LLD, leg length discrepancy; BMI, body mass index. 
Table 2 Difference of Variables on Both Sides

\begin{tabular}{|l|l|l|l|}
\hline Variables & No Perception on LLD & Perception on LLD & $P$-values \\
\hline Difference in FL & $10.5 \pm 7.7$ & $11.0 \pm 8.3$ & 0.807 \\
\hline Difference in TL & $5.8 \pm 3.8$ & $5.2 \pm 4.4$ & 0.474 \\
\hline Difference in TD-TP & $6.5 \pm 4.3$ & $10.3 \pm 7.5$ & 0.018 \\
\hline Difference in CH-TP & $9.9 \pm 7.5$ & $12.5 \pm 9.7$ & 0.412 \\
\hline Difference in GT-TP & $19.1 \pm 11.3$ & $19.3 \pm 13.4$ & 0.958 \\
\hline Difference in FL + TL & $6.5 \pm 4.7$ & $10.7 \pm 7.5$ & 0.009 \\
\hline
\end{tabular}

Abbreviations: LLD, leg length discrepancy; FL, femoral length; TL, tibial length; $\mathrm{CH}$, center of femoral head; TP, the midpoint of tibial plafond; TD, inferior aspect of teardrop; GT, apex of greater trochanter.

Table 3 Results of Multiple Logistic Regression

\begin{tabular}{|l|l|l|l|}
\hline Variables & Adjusted Odds Ratio* & 95\% Confidence Interval & $P$-values \\
\hline Difference in TD-TP & 1.157 & $1.022-1.310$ & 0.021 \\
\hline Difference in CH-TP & 1.038 & $0.973-1.107$ & 0.263 \\
\hline Difference in GT-TP & 0.996 & $0.953-1.042$ & 0.870 \\
\hline Difference in FL+TL & 1.166 & $1.035-1.315$ & 0.012 \\
\hline
\end{tabular}

Note: *The value was adjusted for gender, age, body mass index, and side.

Abbreviations: FL, femoral length; TL, tibial length; $\mathrm{CH}$, center of femoral head; TP, the midpoint of tibial plafond; TD, inferior aspect of teardrop; GT, apex of greater trochanter.

between the patients with no perception on LLD and the patients with perception on LLD. In multiple logistic regression (Table 3), no correlation was found among the perception on LLD and the difference in CH-TP $(P=0.263)$, and the difference in GT-TP $(P=0.870)$. Association was found among the patients' perception on LLD with difference in TD-TP (adjusted odds ratio $=1.157$, $P=0.021$ ), and the difference in FL+TL (adjusted odds ratio=1.166, $P=0.012$ ).

The ROC curves (Figure 2) showed that the area under the curve (AUC) of the difference in FL+TL and the difference TD-TP $(0.704 ; 95 \% \quad \mathrm{CI}=0.562-0.845$ and $0.679 ; 95 \% \mathrm{CI}=0.541-0.817$, respectively) were significantly higher than those of the difference in CH-TP and the difference in GT-TP $(0.564 ; 95 \%$ confidence interval $=0.403-0.721$ and $0.483 ; 95 \%$ confidence interval $=0.332-0.845$, respectively). With the calculated threshold of LLD set at $9.0 \mathrm{~mm}$, the sensitivity and specificity of the difference in TD-TP and the difference in FL +TL were $57.7 \%, 79.4 \%$ and $61.5 \%, 79.4 \%$, respectively.

Both the intraobserver and the interobserver agreement were found to be nearly perfect for all of the measurements (Table 4).

\section{Discussion}

This retrospective study investigated the correlation between the patients' perception on LLD and different variables measured in the full-length standing anteroposterior radiographs after primary THA. In multiple logistic regression and the ROC curves, perceived LLD was associated with the difference in TD-TP and the difference in FL+TL. The sensitivity and specificity of the difference in TD-TP and the difference in FL+TL were 57.7\%, 79.4\% and $61.5 \%, 79.4 \%$, respectively when the calculated thresholds of the difference in TD-TP and the difference in FL+TL were set at $9.0 \mathrm{~mm}$.

The pelvic radiograph is widely used for LLD detection in clinical practice because of its simplicity and its low radiation exposure. Patients with unilateral DDH may present with LLD derived from both the femur and the tibial. $^{14,15}$ Using the pelvic radiograph to predict LLD is not reliable. Zhang et $\mathrm{al}^{14}$ recommended that the use of full-length standing anteroposterior radiographs for LLD detection is advisable for patients with DDH because of its good accuracy and reliability.

The center of the hip is used as a landmark for LLD measurement in the full-length standing anteroposterior 


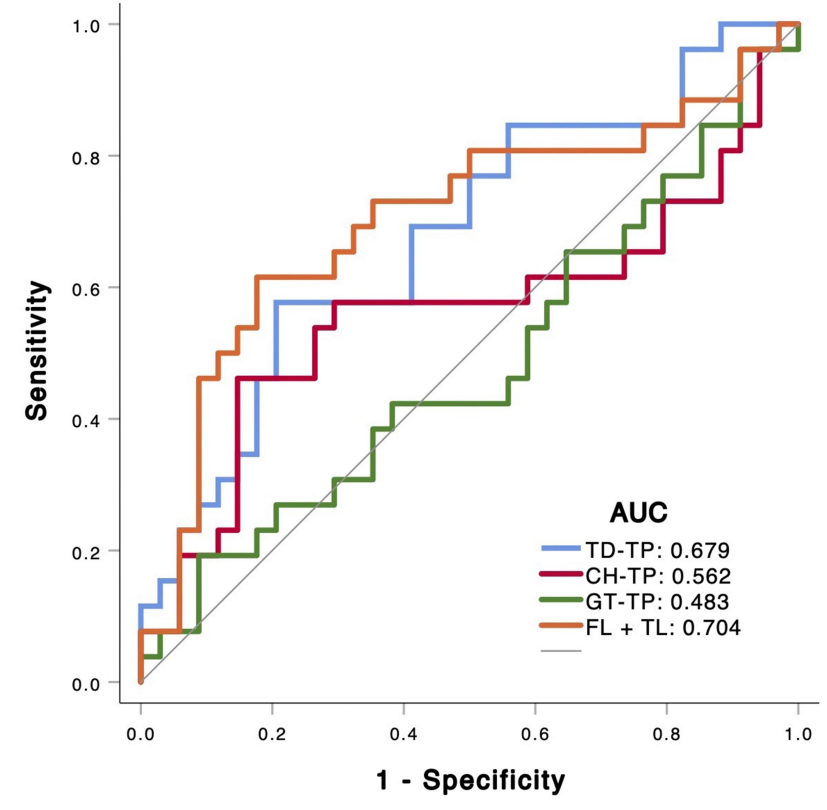

Figure 2 The ROC curves for each measurement for radiographic LLD. Patients perception on LLD had good correlation and reliability on the difference of FL+TL and the difference of TD-TP on both sides in the full-length standing anteroposterior radiographs.

Abbreviations: $L L D$, leg length discrepancy; $F L+T L$, the sum of femoral length and tibial length; TD-TP, the distance between the inferior aspect of teardrop and the midpoint of tibial plafond.

radiographs. $^{6-8}$ However, the center of the hip in the operative side may be affected by the position of the acetabular shell. For the patients with Crowe type IV $\mathrm{DDH}$ in our institute, the acetabular shell was located in the posterior and inferior position of the true acetabulum. ${ }^{11}$ The height of the center of the acetabular shell in the operative side was lower than that in the contralateral side, which may cause the difference of CH-TP to be inconsistent with the patients' perception on LLD. Many studies also confirmed the patients' perception on the difference of $\mathrm{CH}-\mathrm{TP}$ had poor correlation and reliability in primary THA. $^{7,8}$
In our study, the patients' perception on the difference of GT-TP also had also poor correlation and reliability. GT-TP was influenced by many factors, such as the SSTO and the femorotibial angle. In the setting of Crowe type IV DDH, SSTO may be necessary to safely reduce the hip to the true acetabulum, mitigate hip soft-tissue contractions, and protect the neurovascular structures. ${ }^{10,16}$ The GT-TP in the operative side after SSTO was significantly shorter than that in the contralateral side. In patients with Crowe type IV DDH, the femoral head was dislocated outwards and upward. In order to keep the leg alignment perpendicular to the ground, the valgus knee deformity was very common in the operative side. Therefore, the difference of GT-TP had poor accuracy and reliability on the true LLD and patients' perception on LLD.

As described in most literature, LLD is defined as the difference of the distance between a femoral and a pelvic landmark on both sides. ${ }^{3,17}$ As a pelvic reference, the teardrop is used in many studies. ${ }^{13,17}$ Because the teardrop is less affected by the position of the pelvis, it is more reliable than other pelvic landmarks. In our study, we found the difference of the TD-TP and the difference of FL+TL had good correlation and reliability on patients' perception. Sectional measurement of the sum of FL and $\mathrm{TL}$ had a fair performance $(\mathrm{AUC}=0.704)$ in the patients' perception on LLD. However, the AUC of the difference of the TD-TP was only 0.679 . The difference may be caused by valgus knee deformity in the operative side.

There is a broad consensus that less than $10 \mathrm{~mm}$ of LLD on radiographs is clinically acceptable. ${ }^{18,19}$ It also had been demonstrated previously that $10 \mathrm{~mm}$ inequality results in activation of compensatory mechanisms, such as a functional scoliosis or contraction of gluteus medius, which continues to increase in proportion to the imposed LLD. ${ }^{18,20}$ Lawrence $^{21}$ proposed that no alterations in body posture or mechanics were activated at LLD of $6 \mathrm{~mm}$ and below. The study results in a pelvic radiograph of Fujita

Table 4 Intraobserver and Interobserver Variations of Measurements

\begin{tabular}{|l|l|l|}
\hline Variables & Intraobserver (ICC) & Interobserver (ICC) \\
\hline Difference in TD-TP & 0.89 & 0.85 \\
\hline Difference in CH-TP & 0.85 & 0.84 \\
\hline Difference in GT-TP & 0.88 & 0.86 \\
\hline Difference in FL+TL & 0.92 & 0.90 \\
\hline
\end{tabular}

Abbreviations: ICC, intraclass correlation coefficient; FL, femoral length; TL, tibial length; $\mathrm{CH}$, center of femoral head; TP, the midpoint of tibial plafond; TD, inferior aspect of teardrop; GT, apex of greater trochanter. 
et $\mathrm{al}^{13}$ show that $7 \mathrm{~mm}$ may be a reasonable threshold for reducing the residual discomfort. In our study, LLD of $9.0 \mathrm{~mm}$ may be a cutoff value to assess whether the patients' perception on LLD, and the sensitivity and specificity of the difference in FL+TL and the difference in TD-TP were $57.7 \%, 79.4 \%$ and $61.5 \%, 79.4 \%$, respectively.

The limitations of the study were 1) no data of preoperative LLD that might influence postoperative patient's perception, and 2) no data of pelvic obliquity that was important for the postoperative patient's perception on LLD. Zhang et $\mathrm{al}^{22}$ found the pelvic obliquity changes significantly in the first year after THA in patients with DDH. Therefore, we selected the patients with at least 12 months after THA, in order to reduce the influence of pelvic obliquity on LLD.

\section{Conclusion}

This study showed that patients' perception on LLD had good correlation and reliability on the difference of FL $+\mathrm{TL}$ and the difference of TD-TP on both sides in the fulllength standing anteroposterior radiographs at least 12 months after THA in patients with unilateral Crowe type IV DDH. The calculated threshold of the difference in FL $+\mathrm{TL}$ and the difference in TD-TP was set at $9.0 \mathrm{~mm}$ to assess the patients' perception on LLD.

\section{Ethics Approval and Informed Consent}

This retrospective study involving human participants was in accordance with the ethical standards of the institutional and national research committee and with the 1964 Helsinki Declaration and its later amendments or comparable ethical standards. The Ethics Committee of our hospital, General Hospital of Chinese People's Liberation Army, approved the study protocol. All the study participants provided written informed consent for the study.

\section{Consent for Publication}

Written informed consent was obtained from all patients for publication of this study and any accompanying images.

\section{Author Contributions}

All authors made substantial contributions to the conception and design, acquisition of data, or analysis and interpretation of data; took part in drafting the article or revising it critically for important intellectual content; gave final approval of the version to be published; and agree to be accountable for all aspects of the work.

\section{Disclosure}

The authors declare that they have no conflicts of interest.

\section{References}

1. Li Y, Zhang X, Wang Q, et al. Equalisation of leg lengths in total hip arthroplasty for patients with Crowe type-IV developmental dysplasia of the hip: classification and management. Bone Joint J. 2017;99-b (7):872-879. doi:10.1302/0301-620X.99B7.BJJ-2016-1328.R1

2. Zhang Y, Chang F, Wang C, Yang M, Wang J. Pelvic reference selection in patients with unilateral Crowe type IV DDH for measuring leg length inequality. Hip Int. 2015;25(5):457-460. doi:10.5301/ hipint. 5000235

3. Meermans G, Malik A, Witt J, Haddad F. Preoperative radiographic assessment of limb-length discrepancy in total hip arthroplasty. Clin Orthop Relat Res. 2011;469(6):1677-1682. doi:10.1007/s11999-0101588-X

4. Erdem Y, Bek D, Atbasi Z, Neyisci C, Yildiz C, Basbozkurt M. Total hip arthroplasty with rectangular stems and subtrochanteric transverse shortening osteotomy in Crowe type IV hips: a retrospective study. Arthroplast Today. 2019;5(2):234-242. doi:10.1016/j. artd.2019.03.002

5. Li X, Lu Y, Sun J, Lin X, Tang T. Treatment of crowe type-IV hip dysplasia using cementless total hip arthroplasty and double chevron subtrochanteric shortening osteotomy: a 5- to 10-year follow-up study. J Arthroplasty. 2017;32(2):475-479. doi:10.1016/j.arth.201 6.07 .050

6. Lecoanet P, Vargas M, Pallaro J, Thelen T, Ribes C, Fabre T. Leg length discrepancy after total hip arthroplasty: can leg length be satisfactorily controlled via anterior approach without a traction table? Evaluation in 56 patients with EOS 3D. Orthop Traumatol Surg Res. 2018;104(8):1143-1148. doi:10.1016/j.otsr.2018.06.020

7. Piyakunmala K, Sangkomkamhang T. Measurement of patient's perception on limb-length discrepancy compared with weight-bearing orthoroentgenography in total hip arthroplasty: a prospective study. $J$ Arthroplasty. 2018;33(7):2301-2305. doi:10.1016/j.arth.201 8.02 .024

8. Lazennec JY, Folinais D, Florequin C, Pour AE. Does patients' perception of leg length after total hip arthroplasty correlate with anatomical leg length? J Arthroplasty. 2018;33(5):1562-1566. doi:10.1016/j.arth.2017.12.004

9. Woolson ST, Hartford JM, Sawyer A. Results of a method of leg-length equalization for patients undergoing primary total hip replacement. $J$ Arthroplasty. 1999;14(2):159-164. doi:10.1016/ S0883-5403(99)90119-5

10. Wang S, Zhou Y, Ma H, Du Y, Piao S, Wu W. Mid-term results of total hip replacement with subtrochanteric osteotomy, modular stem, and ceramic surface in Crowe IV hip dysplasia. Arthroplast Today. 2018;4(3):363-369. doi:10.1016/j.artd.2017.07.003

11. Zhou Y, Sun C, Wang Y. New method addressing the problem of using ceramic-on-ceramic bearing in too small acetabulum of high-riding DDH patients with THA. Semin Arthroplasty. 2012;23 (4):226-231. doi:10.1053/j.sart.2012.12.006

12. Du YQ, Sun JY, Ma HY, Wang S, Ni M, Zhou YG. Leg length balance in total hip arthroplasty for patients with unilateral crowe type IV developmental dysplasia of the hip. Orthop Surg. 2020. doi:10.1111/os.12667

13. Fujita K, Kabata T, Kajino Y, Tsuchiya H. Optimizing leg length correction in total hip arthroplasty. Int Orthop. 2020;44(3):437-443. doi:10.1007/s00264-019-04411-0 
14. Zhang Z, Luo D, Cheng H, Xiao K, Zhang H. Unexpected long lower limb in patients with unilateral hip dislocation. J Bone Joint Surg Am. 2018;100(5):388-395. doi:10.2106/JBJS.17.00187

15. Tamura K, Takao M, Hamada H, Ando W, Sakai T, Sugano N. Femoral morphology asymmetry in hip dysplasia makes radiological leg length measurement inaccurate. Bone Joint J. 2019;101-b (3):297-302. doi:10.1302/0301-620X.101B3.BJJ-2018-0965.R1

16. Greber EM, Pelt CE, Gililland JM, Anderson MB, Erickson JA, Peters CL. Challenges in total hip arthroplasty in the setting of developmental dysplasia of the hip. J Arthroplasty. 2017;32(9s): S38-s44.

17. Shi XT, Cheng CM, Feng CY, Li CF, Li SX, Liu JG. Crowe type IV hip dysplasia treated by THA combined with osteotomy to balance functional leg length discrepancy: a prospective observational study. Orthop Surg. 2020;12(2):533-542. doi:10.1111/os.12655

18. O'Brien S, Kernohan G, Fitzpatrick C, Hill J, Beverland D. Perception of imposed leg length inequality in normal subjects. Hip Int. 2010;20(4):505-511. doi:10.1177/112070001002000414
19. Chen G, Nie Y, Xie J, Cao G, Huang Q, Pei F. Gait analysis of leg length discrepancy-differentiated hip replacement patients with developmental dysplasia: a midterm follow-up. J Arthroplasty. 2018;33(5):1437-1441. doi:10.1016/j.arth.2017.12.013

20. Vink P, Kamphuisen HA. Leg length inequality, pelvic tilt and lumbar back muscle activity during standing. Clin Biomech (Bristol, Avon). 1989;4(2):115-117. doi:10.1016/0268-0033(89)90049-1

21. Lawrence D. Lateralization of weight in the presence of structural short leg: a preliminary report. J Manipulative Physiol Ther. 1984;7 (2):105-108.

22. Zhang Y, Cheng T, Zhang XL. Changes in intra-pelvic obliquity angle 0-2 years after total hip arthroplasty and its effects on leg length discrepancy: a retrospective study. Chin Med J. 2015;128 (10):1346-1350. doi:10.4103/0366-6999.156780
Patient Preference and Adherence

\section{Publish your work in this journal}

Patient Preference and Adherence is an international, peer-reviewed, open access journal that focusing on the growing importance of patient preference and adherence throughout the therapeutic continuum. Patient satisfaction, acceptability, quality of life, compliance, persistence and their role in developing new therapeutic modalities and compounds to optimize clinical outcomes for existing disease

\section{Dovepress}

states are major areas of interest for the journal. This journal has been accepted for indexing on PubMed Central. The manuscript management system is completely online and includes a very quick and fair peer-review system, which is all easy to use. Visit http:// www.dovepress.com/testimonials.php to read real quotes from published authors. 